\title{
Eficácia da realidade virtual sobre o equilíbrio de idosos
}

\author{
Effectiveness of virtual reality on the balance in the elderly \\ Eficacia de la realidad virtual en el equilibrio en los ancianos \\ Yago Tavares PINHEIRO ${ }^{1}$ \\ Dayvianne Cecília Ribeiro Moreira TEIXEIRA ${ }^{1}$ \\ Rodolfo de Medeiros MENDONÇA ${ }^{2}$ \\ Bárbara Helena Ferreira FREIRE ${ }^{2}$ \\ Bruno Henrique Ferreira FREIRE ${ }^{2}$ \\ Laura de Sousa Gomes VELOSO \\ ${ }^{1}$ Fisioterapeuta pela Faculdade Maurício de Nassau, Av. Pres. Epitácio Pessoa, 1201 - Bairro dos Estados, \\ 58030-000, João Pessoa-PB, Brasil \\ ${ }^{2}$ Acadêmico de Fisioterapia, Faculdade Maurício de Nassau, Av. Pres. Epitácio Pessoa, 1201 - Bairro dos Estados, \\ 58030-000, João Pessoa-PB, Brasil \\ ${ }^{3}$ Fisioterapeuta, Mestranda em Enfermagem, Universidade Federal da Paraíba UFPB \\ Professora do Curso de Fisioterapia, Faculdade Maurício de Nassau, Av. Pres. Epitácio Pessoa, 1201 - Bairro dos Estados, \\ 58030-000, João Pessoa-PB, Brasil
}

\begin{abstract}
Resumo
Introdução: A prevalência de quedas tem se configurado na atual conjuntura como um problema de saúde pública, tendo em vista que está relacionado às principais causas de morte de idosos. Assim, surge a realidade virtual (gameterapia) como ferramenta bastante utilizada na atualidade para a reabilitação e prevenção de quedas nessa população. Objetivo: avaliar a eficácia da realidade virtual (gameterapia) sobre o equilíbrio de idosos. Metodologia: Trata-se de uma revisão bibliográfica a partir de estudos do tipo ensaio clínico e caso-controle, indexados nas bases de dados LILACS, SCIELO e MEDLINE, publicados em inglês, português e espanhol, entre 2010 e 2015. A busca se procedeu nas referidas bases de dados pelo cruzamento livre dos descritores "jogos de vídeo" (vídeo game), "equilíbrio postural" (postural balance) e "idoso" (aged). Resultados: 11 estudos se enquadraram rigorosamente nos critérios de elegibilidade e, portanto, foram inclusos na presente revisão. Todos os estudos abordavam a questão da realidade virtual, na maioria das vezes associada a outras terapias para melhora de equilíbrio. Um achado comum a grande maioria foi a melhora nos índices de equilíbrio e redução do risco de quedas nos grupos em que se praticou a técnica. Conclusão: A gameterapia é um recurso que deve ser utilizado como maneira alternativa nos tratamentos fisioterapêuticos, pois se mostra tanto um recurso eficaz quanto um meio de estimulo ao paciente idoso, que se sente atraído pela dinâmica dos games de realidade virtual, o que os leva a uma aceitação e adesão mais rápida ao tratamento.

Descritores: Jogos de vídeo; Equilíbrio postural; Idoso.
\end{abstract}

\begin{abstract}
Introduction: The prevalence of falls has been set in the current situation as a public health problem, with a view that is related to the leading causes of death among the elderly. Thus, virtual reality arises (game therapy) as a tool widely used today for the rehabilitation and prevention of falls in this population. Objective: To evaluate the effectiveness of virtual reality (game therapy) on the balance of the elderly. Materials and methods: This is a literature review from studies of clinical trial type and casecontrol indexed in the databases LILACS, SciELO and MEDLINE, published in English, Portuguese and Spanish between 2010 and 2015. The search was carried in such databases by the free crossing of descriptors "video games" (video game), "postural balance" (postural balance) and "old" (aged). Results: 11 studies not fit strictly the eligibility criteria and therefore were included in this review. All studies addressed the issue of virtual reality, most often associated with other therapies for improved balance. A common finding the most was the improvement in the balance of rates and reducing the risk of falls in the groups that practiced technique. Conclusion: game therapy is a resource that should be used as an alternative way in the physical therapy treatments, because it shows both an effective remedy as a means of stimulating the elderly patient, who is attracted by the dynamics of virtual reality games, which takes to faster acceptance and adherence to treatment.

Descriptors: Video Games; Postural Balance; Aged.
\end{abstract}

\section{Resumen}

Introducción: La prevalencia de caídas se ha puesto en la situación actual como un problema de salud pública, con el fin de que se relaciona con las principales causas de muerte entre los mayores. Por lo tanto, surge la realidad virtual (terapia con videojuegos) como una herramienta ampliamente utilizada en la actualidad para la rehabilitación y la prevención de caídas en esta población. Objetivo: Evaluar la eficacia de la realidad virtual (terapia con videojuegos) en el equilibrio de los ancianos. Materiales y métodos: Se trata de una revisión de la literatura de los estudios de tipo ensayo clínico y de casos y controles indexados en las bases de datos LILACS, SciELO y MEDLINE, publicados en Inglés, portugués y español entre 2010 y 2015. La búsqueda se realizó en estas bases por el paso libre de los descriptores "videojuegos" (videojuegos), "el equilibrio postural" (equilibrio postural) y "viejo" (años de edad). Resultados: 11 estudios no se ajustan estrictamente los criterios de elegibilidad y, por tanto, se incluyeron en esta revisión. Todos los estudios se abordaron la cuestión de la realidad virtual, lo más a menudo asociados con otras terapias para la mejora del equilibrio. Un hallazgo común más fue la mejora en el equilibrio de los precios y la reducción del riesgo de caídas en los grupos que practicaron la técnica. Conclusión: terapia con videojuegos es un recurso que debe ser utilizado como una forma alternativa en los tratamientos de terapia física, ya que muestra tanto un recurso efectivo como medio de estimular el paciente anciano, que se siente atraída por la dinámica de juegos de realidad virtual, que toma a la aceptación más rápida y adherencia al tratamiento.

Descriptores: Juegos de Video; Balance Postural; Anciano.

\section{INTRODUÇÃO}

O mundo tem experimentado nas últimas décadas uma mudança nos padrões etários populacionais que se configura na forma de envelhecimento da população, antes experimentado apenas em países desenvolvidos como os da Europa e, atualmente, se estendendo àqueles emergentes, a exemplo do Brasil. Estima-se que até 2025, mundialmente, 
exista 1,2 bilhão de pessoas com mais de 60 anos de idade. No Brasil, como prova da atenuação desse processo de envelhecer, há evidências que mostram um aumento de $500 \%$ no número de idosos, que cresceu de 3 milhões em 1960 para 14 milhões em 2002. Além disso, presume-se que esse número chegue à casa dos 32 milhões em $2020^{1,2}$.

Esse processo, resultado da diminuição da taxa de fecundidade e de mortalidade, é biologicamente descrito como sendo um retrocesso morfológico e funcional de natureza multifatorial que afeta de maneira negativa todos os sistemas do corpo e seu funcionamento próprio, levando progressivamente a um declínio das funções fisiológicas e consequente diminuição da capacidade funcional ${ }^{3}$.

O envelhecer traz consigo algumas alterações, entre elas, as dos sinais vestibulares, visuais e proprioceptivos, além da diminuição dos reflexos de proteção, responsáveis pelo equilíbrio e controle motor que, por sua vez, é definido como a capacidade de manter o corpo estabilizado no espaço $^{4,5}$.

A partir do momento em que o indivíduo teve seu balanço postural alterado por fatores intrínsecos ou extrínsecos, de caráter senil ou senescente, este irá sofrer de desequilíbrio que culminará no aumento do risco de quedas que tem se configurado na atual conjuntura como um problema de saúde pública, tendo em vista que está relacionado às principais causas de morte, seja de forma direta ou indireta, de idosos ${ }^{4}$.

Como ferramenta para terapêutica dos déficits de equilíbrio dos idosos, muito tem se falado na gameterapia por causa de seus resultados satisfatórios. Esta se dá por meio da interação entre o indivíduo e o computador em um ambiente, criando-se uma realidade virtual e realista, gerando um feddbackvisual através do vídeo. A cada dia é mais comum o uso da gameterapia por fisioterapeutas com o intuito de habilitar e reabilitar necessidades específicas de pacientes $^{6,7}$.

Sendo assim, percebendo o crescente número de idosos, a ligação do desequilíbrio prevalente nessa população e o aumento do risco de quedas, assim como a necessidade de se estudar as novas tecnologias que surgem para o manejo de tal déficit, o presente estudo se torna relevante e justificável, uma vez que propõe avaliar a eficácia da realidade virtual (gameterapia) sobre o equilíbrio de idosos.

\section{MATERIAL E MÉTODO}

Trata-se de um estudo exploratório e observacional do tipo revisão de literatura feita nas bases de dados: LILACS (Literatura Latino-Americana e do Caribe em Ciências da Saúde), MEDLINE (Medical Literature Analysisand Retrieval System Online) e SCIELO (Scientific Electronic Library Online), a partir do cruzamento dos descritores: Jogos de vídeo (vídeo game), equilíbrio postural (postural balance) e idoso (aged).

Inicialmente os documentos encontrados sofreram uma primeira filtragem com base na leitura de seus títulos e resumos, a fim de conferir a adequação com a temática proposta. Posteriormente foram submetidos a critérios de inclusão e exclusão estabelecidos pelos pesquisadores e dispostos no quadro 1. Por fim, os manuscritos foram lidos na íntegra para a análise e síntese de suas principais informações.

Com a busca no LILACS foram encontrados 32 estudos, dos quais apenas 01 enquadra-se nos critérios de elegibilidade. Já no MEDLINE, obteve-se 75 estudos achados, sendo 07 selecionados. No SCIELO, por sua vez, de 67 publicações, 03 foram incluídas. Portanto, a presente revisão de literatura foi composta por um total de 11 estudos.

Quadro 1: Critérios de elegibilidade para os estudos encontrados.

\begin{tabular}{|c|c|}
\hline Critérios de inclusão & Critérios de exclusão \\
\hline $\begin{array}{l}\text { - Artigos indexados nas bases } \\
\text { de dados LILACS, MEDLINE e } \\
\text { SCIELO; } \\
\text { - Artigos do tipo ensaio clínico e } \\
\text { caso-controle; } \\
\text { - Artigos publicados entre janeiro } \\
\text { de } 2010 \text { e dezembro de } 2015 ; \\
\text { - Artigos escritos em inglês, } \\
\text { português e espanhol; } \\
\text { - Estudo com amostras de idade } \\
\text { igual ou superior a } 60 \text { anos. }\end{array}$ & $\begin{array}{l}\text { - Teses, dissertações, } \\
\text { monografias e revisões de } \\
\text { literatura; } \\
\text { - Artigos que não } \\
\text { contemplavam o objeto e } \\
\text { a temática proposta. }\end{array}$ \\
\hline
\end{tabular}

Fonte: dados da pesquisa, 2016.

\section{RESULTADOS}

A presente revisão integrativa analisou 11 artigos científicos que atenderam rigorosamente os critérios de inclusão. Para a síntese dos estudos foi criado com guia de integração (Quadro 2) contendo informações sobre título e autor, ano e base de dados em que foi publicado, objetivo principal, tipo e abordagem do estudo, metodologia adotada e resultados.

Todos os estudos abordavam a questão da realidade virtual, na maioria das vezes associada a outras terapias para melhora de equilíbrio. Um achado comum a grande maioria foi a melhora nos índices de equilíbrio e redução do risco de quedas nos grupos em que se praticou a técnica em estudo.

\section{DISCUSSÃO}

Sabe-se que o controle postural é resultado de uma interação complexa dos sistemas sensoriais (visual, vestibular e somatossensorial), do sistema motor e do sistema nervoso central. Pesquisas com idosos saudáveis mostram um padrão de disparidade na utilização de informações sensoriais e motoras como consequência do processo de envelhecimento, demonstrando que as alterações desses sistemas causam alterações do equilíbrio ${ }^{8}$.

Exercícios preventivos que visam melhorar a força muscular e controle postural têm sido frequentemente recomendados para adultos mais velhos. Intervenções especialmente computadorizadas parecem ser propícias para este fim ${ }^{9}$.

A realidade virtual é uma tecnologia que permite ao usuário interagir diretamente com um computador simulandor de meio ambiente, em tempo real e/ou em duas ou três dimensões. Essa interface exige do usuário a realização de movimentos discretos, controlados para além de sua base de apoio em resposta a impulsos visuais ${ }^{10}$.

Estudos apontam que há melhoria significativa tanto no equilíbrio dinâmico quanto a confiança em adultos mais velhos ${ }^{11}$.

Segundo pesquisas obtemos uma melhora para velocidade da marcha e apoio simples e equilíbrio corporal, assim diminuindo o risco de quedas em ambiente domiciliar e comunitário, mas quando comparado a métodos tradicionais da fisioterapia não se observa diferenças significativas em termos de resultado entre os grupos avaliados ${ }^{9}$.

E possivel verificar que existe uma variedade muito grande de formas de aplicação dos jogos de realidade virtual, pois os autores pesquisados o usaram não só em comparação 
com diversas outras tecnicas como também em diversas patalogias. Os estudos de Zalecki et. al. ${ }^{10}$, Mhatre et. al. ${ }^{12}$, Pompeu et. al. ${ }^{12}$ e Esculier et. al. ${ }^{14}$ corroboram entre si, onde foram expostas as melhorias obtidas com o uso desta modalidade de terapia tais como melhor equilibrio estático e dinâmico, velocidade da marcha, tempo para levantar e sentar, postura unipodal entre outros em pacientes com Doenca de Parkinson

Quadro 2: Guia de integração dos estudos.

\begin{tabular}{|c|c|c|c|c|}
\hline $\begin{array}{c}\begin{array}{c}\text { Título/Autor/Ano de } \\
\text { publicação/ } \\
\text { Base de dados }\end{array} \\
\end{array}$ & Objetivo & $\begin{array}{l}\text { Tipo de estudo/ } \\
\text { Abordagem }\end{array}$ & Metodologia & Resultados \\
\hline $\begin{array}{l}\text { Clinical feasibility of the Nintendo } \\
\text { WiiT for balance training post-stroke: } \\
\text { a phase II randomized controlled trial } \\
\text { in an inpatient setting } \\
\text { Bower et. al, 2014 } \\
\text { SCIELO }\end{array}$ & $\begin{array}{l}\text { Investigar a viabilidade e a } \\
\text { eficácia potencial do } \\
\text { Nintendo Wii TM para o } \\
\text { equilíbrio de reabilitação } \\
\text { após o AVC }\end{array}$ & $\begin{array}{c}\text { Caso } \\
\text { controle/Qualitativa }\end{array}$ & 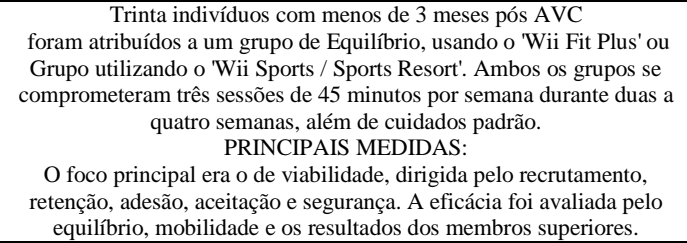 & 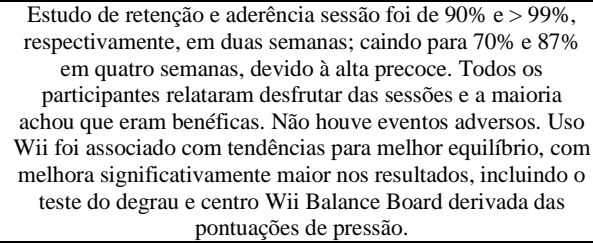 \\
\hline $\begin{array}{l}\text { Virtual-reality balance training with } \\
\text { a video-game system improves } \\
\text { dynamic balance in chronic stroke } \\
\text { patients } \\
\text { Cho et. al., 2012 } \\
\text { SCIELO }\end{array}$ & $\begin{array}{l}\text { Investigar os efeitos do } \\
\text { treinamento de equilíbrio } \\
\text { realidade virtual (VRBT) } \\
\text { com um sistema de jogo de } \\
\text { tabuleiro de equilíbrio na } \\
\text { balança de pacientes com } \\
\text { AVC crônico } \\
\end{array}$ & $\begin{array}{l}\text { Caso controle/ } \\
\text { Quantitativa }\end{array}$ & 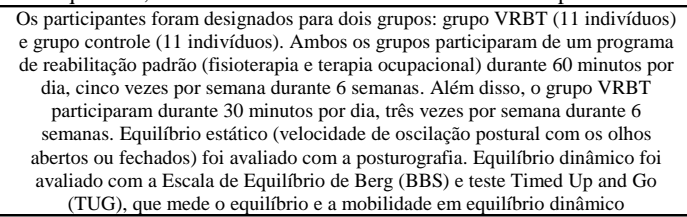 & $\begin{array}{c}\text { Houve melhora maior na BBS (4,00 vs } 2,81 \text { pontos) e TUG (- } \\
1,33 \text { vs }-0,52 \text { sec) no grupo VRBT em comparação com o } \\
\text { grupo controle ( }(\mathrm{P}<0,05) \text {, mas não no equilíbrio estat́tico em } \\
\text { ambos os grupos }\end{array}$ \\
\hline $\begin{array}{l}\text { Home-based balance training } \\
\text { programme using Wii Fit with balance } \\
\text { board for Parkinsons's disease: a pilot } \\
\text { study } \\
\text { Esculier et. al., 2012 } \\
\text { MEDLINE }\end{array}$ & $\begin{array}{l}\text { Avaliar os efeitos de um } \\
\text { programa de treinamento de } \\
\text { equilíbrio usando feedback } \\
\text { visual (Nintendo Wii Fit } \\
\text { jogo com prancha de } \\
\text { equilibrio) no balancono e } \\
\text { funcionais habilidades em } \\
\text { indivividuos com doença de } \\
\text { Parkinson, e comparar os } \\
\text { efeitos com un grupo de } \\
\text { indivíduos saudáveis } \\
\text { emparelhados }\end{array}$ & $\begin{array}{c}\text { Ensaio } \\
\text { clínico/Qualitativa }\end{array}$ & 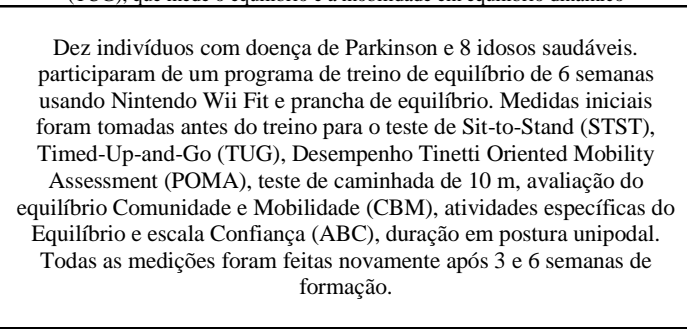 & $\begin{array}{l}\text { A doença de Parkinson melhorou significativamente seus } \\
\text { resultados em TUG, STST, postura unipodal, teste de } 10 \mathrm{~m} \text { de } \\
\text { caminhada, CBM, POMA e plataforma de força no final do } \\
\text { programa de treinamento de } 6 \text { semanas. O grupo de } \\
\text { indivíduos saudáveis melhorou significativamente no TUG, } \\
\text { STST, postura unipodal e CBM. }\end{array}$ \\
\hline $\begin{array}{l}\text { Use of an interactive video gaming } \\
\text { program compared with conventional } \\
\text { physiotherapy for hospitalised older } \\
\text { adults: a feasibility trial } \\
\text { Laver et. al., } 2011^{17} \\
\text { MEDLINE }\end{array}$ & $\begin{array}{l}\text { Avaliar a viabilidade de } \\
\text { uma intervenção } \\
\text { fisioterapêutica utilizando } \\
\text { um programa de jogos } \\
\text { interativos em comparação } \\
\text { com a fisioterapia } \\
\text { convencional paraidosos } \\
\text { hospitalizados } \\
\end{array}$ & $\begin{array}{l}\text { Ensaio clínico } \\
\text { randomizado/Quanti- } \\
\text { qualitativa }\end{array}$ & 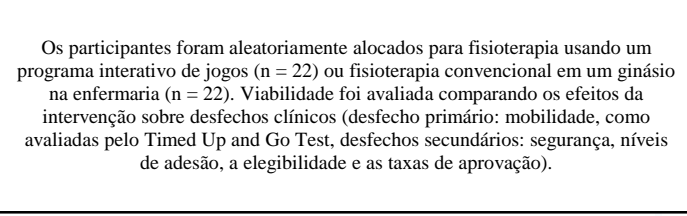 & $\begin{array}{l}\text { Análises univariadas mostraram não haver diferença } \\
\text { significativa entre os grupos após a intervenção. No entanto, } \\
\text { as análises multivariadas sugeriram que os participantes } \\
\text { usando o programa de jogos interativos melhoraram mais no } \\
\text { Timed Uu and Go }(\mathrm{p}=0,048) \text { do que os participantes que } \\
\text { receberam fisioterapia convencional. Não houve eventos } \\
\text { adversos graves e elevados níveis de adesão à terapêeutica } \\
\text { foram evidentes em ambos os grupos. }\end{array}$ \\
\hline $\begin{array}{l}\text { Nintendo Wii e seu efeito no } \\
\text { equilíbrio e capacidade funcional de } \\
\text { idosos saudáveis. } \\
\text { Mussato et. al., 2012 } \\
\text { LILACS }\end{array}$ & $\begin{array}{l}\text { Analisar o efeito do vídeo } \\
\text { game Wii Fit da Nintendo@ } \\
\text { sobre o equilibrio e a } \\
\text { marcha de idosos saudáveis }\end{array}$ & $\begin{array}{l}\text { Caso controle/ } \\
\text { Quantitativa }\end{array}$ & $\begin{array}{l}\text { Participaram } 10 \text { idosos, os quais foram distribuídos em dois grupos: } \\
\text { grupo experimental (GE) e grupo controle (GC). Foram avaliados: o } \\
\text { equilíbrio pela estabilometria, utilizando-se um baropodômetro } \\
\text { eletrônico; o tempo de apoio unipodal e a capacidade funcional pelo } \\
\text { teste Timed up and Go. Antes e eapós o protocolo de treinamento com o } \\
\text { game Wii Fit da Nintendo@, que consistiu de } 10 \text { sessões, com duração } \\
\text { de } 30 \text { minutos cada. }\end{array}$ & 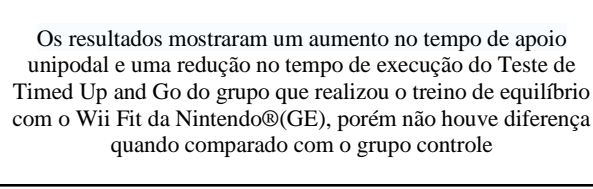 \\
\hline $\begin{array}{l}\text { A cognitive-motor intervention using a } \\
\text { dance video game to enhance foot } \\
\text { placement accuracy and gait under } \\
\text { dual task conditions in older adults: a } \\
\text { randomized controlled trial } \\
\text { Pichierri et. al., 20129 } \\
\text { MEDLINE }\end{array}$ & $\begin{array}{l}\text { Comparar dois grupos de } \\
\text { treinamento com exercícios } \\
\text { de força e equilíbrio por } \\
\text { meio de jogos de vídeo e } \\
\text { dança }\end{array}$ & $\begin{array}{c}\text { Caso } \\
\text { controle/Quantitativa }\end{array}$ & $\begin{array}{l}\text { Moradores foram designados para dois grupos: grupo dança (casos, } \\
\mathrm{n}=15 \text { ) e grupo de controlo }(\mathrm{n}=16) \text {. O grupo de dança foi submetido a } \\
\text { um programa de } 12 \text { semanas de cognitivo-motor duas vezes por } \\
\text { semana que compunham a força progressiva e treinamento de } \\
\text { equilibrio complementado com jogos de vídeo dança adicional. } \mathrm{O} \\
\text { grupo controle realizado apenas os exerćícios de força e equilíbrio } \\
\text { durante este período }\end{array}$ & $\begin{array}{l}\text { A comparacãão entre os grupos revelou diferenças } \\
\text { significativas para velocidade da marcha e para o tempo de } \\
\text { apoio simples durante a condição de dupla tarefa rápida } \\
\text { andando em favor do grupo de dançą. }\end{array}$ \\
\hline $\begin{array}{c}\text { Pilot study comparing changes in } \\
\text { postural control after training using a } \\
\text { video game balance board program } \\
\text { and } 2 \text { standard activity-based balance } \\
\text { intervention programs } \\
\text { Pluchino et. al., 2012 } \\
\text { MEDLINE } \\
\end{array}$ & $\begin{array}{l}\text { Comparar os impactos de } \\
\text { Tai Chi, de um programa de } \\
\text { exercício de equilíbrio } \\
\text { padrão e de programa de } \\
\text { realidade virtual no controle } \\
\text { postural e quedas risco }\end{array}$ & $\begin{array}{l}\text { Caso controle/ } \\
\text { Quantitativa }\end{array}$ & $\begin{array}{l}27 \text { idosos foram submetidos a Tai Chi, um programa de equilíbrio } \\
\text { exerćício padrão, e de programa de realidade virtual e avaliados com as } \\
\text { seguintes medidas: Timed Up \& Go, One--Leg Stance, alcance } \\
\text { funcional, Desempenho Tinetti Oriented Mobility Assessment, } \\
\text { posturografia dinâmica (DP) e risco de quedas }\end{array}$ & $\begin{array}{l}\text { O videogames foi tão eficaz quanto o Tai Chi eo programa de } \\
\text { exercícios de equilibrio padrão para melhorar o controle } \\
\text { postural e o equilibrio }\end{array}$ \\
\hline $\begin{array}{l}\text { Feasibility, safety and outcomes of } \\
\text { playing Kinect Adventures!â,, for } \\
\text { people with Parkinson's disease: a } \\
\text { pilot study } \\
\text { Pompeu et. al., } 2014^{13} \\
\text { MEDLINE }\end{array}$ & $\begin{array}{l}\text { Avaliar a viabilidade, } \\
\text { segurança e resultados de } \\
\text { jogar Microsoft Kinect } \\
\text { Adventuresâ para as pessoas } \\
\text { com a doença de Parkinson }\end{array}$ & $\begin{array}{c}\text { Ensaio } \\
\text { clinico/Qualitativa }\end{array}$ & $\begin{array}{l}\text { Sete pacientes com doença de Parkinson submetidos a catorze sessões } \\
\text { de } 60 \text { minutos, três vezes por semana, jogando quatro jogos de Kinect } \\
\text { Adventures. Os resultados de viabilidade e de segurança foram o } \\
\text { desempenho do jogo dos pacientes e de eventos adversos, } \\
\text { respectivamente. Os resultados clínicos foram o teste de caminhada de } \\
6 \text { minutos, Equilíbrio Avaliação Sistema de Teste, Dynamic Gait Index } \\
\text { e Doença de Parkinson Questionnaire (PDQ-39) }\end{array}$ & 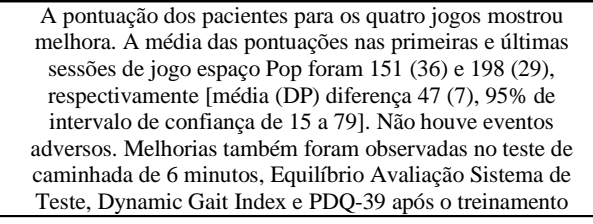 \\
\hline $\begin{array}{l}\text { The effect of virtual reality gaming on } \\
\text { dynamic balance in older adults } \\
\text { Rendon et. al., 201211 } \\
\text { MEDLINE }\end{array}$ & $\begin{array}{l}\text { Comparar um grupo de } \\
\text { realidade virtual (VRG) } \\
\text { um grupo controle (GC) no } \\
\text { tocante ao equilíbrio }\end{array}$ & $\begin{array}{c}\text { Caso } \\
\text { controle/Quantitativa }\end{array}$ & $\begin{array}{l}40 \text { idosos foram divididos em dois grupos: grupo de realidade virtual - } \\
\text { GVR - (casos) e o grupo controle - GC - (sem intervenção). O GVR } \\
\text { Recebeu três diferentes intervenções foi trabalhado com realidade } \\
\text { virtual por6 semanas, enquanto o grupo controle não recebeu nenhuma } \\
\text { intervenção. }\end{array}$ & $\begin{array}{l}\text { Em comparação com o GC, as mediç̃oes pós-intervenção } \\
\text { mostraram melhorias significativas para a VRG no Up \& Go } \\
\text { teste de } 8 \text { pés ( } \mathrm{p}=0,038) \text {, na Escala de Autoconfiança e no } \\
\text { equilíbrio de atividades específicas }(\mathrm{p}=0,038)\end{array}$ \\
\hline $\begin{array}{l}\text { Visual feedback training using WII Fit } \\
\text { improves balance in Parkinson's } \\
\text { disease } \\
\text { Zalecki et. al., 2013 } \\
\text { MEDLINE }\end{array}$ & $\begin{array}{l}\text { Investigar a eficácia de um } \\
\text { método de treinamento de } \\
\text { feedback visual-novela, } \\
\text { usando Wii Fit balance } \\
\text { bard na melhora do } \\
\text { equilibrio em pacientes com } \\
\text { DP }\end{array}$ & $\begin{array}{l}\text { Ensaio clínico/Quanti- } \\
\text { qualitativa }\end{array}$ & $\begin{array}{l}\text { Vinte e quatro pacientes com DP moderada foram incluídos no estudo } \\
\text { que constou de um programa de treinamento de equilíbrio baseado em } \\
\text { casa de } 6 \text { semanas usando Nintendo Wii Fit e prancha de equilibrio }\end{array}$ & $\begin{array}{l}\text { As pacientes melhoraram significativamente os seus } \\
\text { resultados em Berg Balance Scale, orientada para o } \\
\text { desempenho de avaliação da mobilidade do Tinnet, Timed } \\
\text { Up-and-Go, Sit-to-stand teste, 10-Meter teste de caminhada e } \\
\text { atividades específicas escala Confiança }\end{array}$ \\
\hline
\end{tabular}

Em relação aos cuidados com o paciente com sequelas por um Acidente vascular encefálico (AVE), Bower et. al. ${ }^{15}$ realizaram uma comparação entre duas modalidades de gameterapia onde os participantes da pesquisa desfrutavam das sessões e relatavam que as mesmas eram benéficas. Kin Hun Cho et.al. ${ }^{16}$ praticam metodos tradicionais fisioterapêuticos associados à realidade virtual, comprova então que em alguns aspectos como escala de Berg e TUG (Timed To Get Up and Go) houveram melhorias com relação aos participantes que não receberam o incentivo da realidade virtual, porém em equilibrio estático todos os participantes melhoraram.
A gameterapia e o uso de jogos de realidade virtual se mostram por diversas vezes tão eficaz quanto outros métodos tais como o Tai $\mathrm{Chi}^{17}$ e outros métodos já usuais da fisioterapia, quando comparados trazem melhorias a qualidade de vida, auto estima e confiança de individuos idosos senis ou senescentes o que o torna uma maneira ludica e inteligente tanto para reabilitar ou realizar a manutanção do bem estar quanto para divertir e entreter o idoso que precisa também de um estímulo que o leve a gostar do tratamento que está lhe sendo proposto.

Utilizando também em ambiente hospitalar, os escritos de Laver et. al. ${ }^{18}$ trazem resultados tão bons quanto 
os das técnicas convencionais utilizadas com maior frequência, demonstrando uma maior adesão ao tratamento e levando um maior número de idosos a concluí-los.

A terapia se mostra eficaz e atrativa para o tratamento de diversas patologias assim como apenas para manuntenção da homeostase corporal dos individuos submetidos a tal.

A presente pesquisa envolveu 38 artigos sobre a gameterapia e utilização de suas diversas modalidades aplicadas a diversos quadros patologiocos além de enfermos e não patologicos também, trazendo melhora de sua condição atual, melhora na sua qualidade de vida e realização de suas atividades diárias.

\section{CONCLUSÃO}

A gameterapia é um recurso que deve ser levado em consideração para ser utilizado como maneira alternativa nos tratamentos fisioterapêuticos, pois se mostra tanto um recurso eficaz quanto um meio de estimulo ao paciente idoso, que se sente atraído pela dinâmica dos games de realidade virtual, o que os leva a uma aceitação e adesão mais rápida ao tratamento, entre os benefícios atingidos com esta modalidade de tratamento podemos ver o aumento do equilíbrio, da velocidade da marcha, da autoestima, confiança para atividades diárias além de muitos outros, através desta pesquisa pudemos identificar a eficácia da gameterapia que por diversas vezes se equiparou aos métodos tradicionais da fisioterapia e até mesmo a métodos como o Tai Chi.

\section{REFERÊNCIAS}

1. World Health Organization. Envelhecimento ativo: uma política de saúde / World Health Organization; tradução Suzana Gontijo. - Brasília: Organização Pan-Americana da Saúde; 2005.

2. Lima-Costa MF, Veras R. Saúde pública e envelhecimento. Cad Saúde Pública. 2003; 19(3):700-1.

3. Moraes EN, Moraes FL, Lima SPP. Características biológicas e psicológicas do envelhecimento. Rev Med Minas Gerais. 2010;20(1):67-73.

4. Ruwer SL, Rossi AG, Simon LF. Equilíbrio no idoso. Rev Bras Otorrinolaringol. 2005; 71(3);298-303.

5. Gazzola, J. M.; Muchale, S. M.; Perracini, M. R.; Cordeiro, R. C.; Ramos, L. R. (2004). Caracterização funcional do equilíbrio de idosos em serviço de reabilitação gerontológica. Rev Fisioter Univ São Paulo. 2004; 11(1):1-14

6. Pimentel MM, Taveira RS, Silva KCO, David MCMM, Franco CIF. Influência da gameterapia sobre o equilíbrio de portadores de doença de Parkinson. In: Anais do $4^{\circ}$ Congresso Internacional de Envelhecimento. 2015;2(1):101-7.

7. Balista VG. Sistema de Realidade Virtual para Avaliação e Reabilitação de Déficit Motor. In: Proceedings do XII Simpósio Brasileiro de Games e Entretenimento Digital; 2013:16-18.

8. Mussato, R.; Brandalize, D.; Brandalize, M. Nitendo wii e seu efeito no equilíbrio e capacidade funcional de idosos saudáveis. R bras ci e mov. 2012; 20(2):68-75

9. Pichierri G, Coppe A, Lorenzetti S, Murer K, de Bruin ED. The effect of a cognitive-motor intervention on voluntary step execution under single and dual task conditions in older adults: a randomized controlled pilot study. J Clin Interv Aging.2012;7(1):175-84.

10. Zalecki T, Gorecka-Mazur A, Pietraszko W, Surowka AD, Novak P, Moskala $M$, et al. Visual feedback training using WII Fit improves balance in Parkinson's disease. Folia Med Cracov. 2013; 53(1):65-78.

11. Rendon AA, Lohman EB, Thorpe D, Johnson EG, Medina E, Bradley B. The effect of virtual reality gaming on dynamic balance in older adults. Age Ageing. 2012;41(4):549-52.

12. Mhatre PV, Vilares I, Stibb SM, Albert MV, Pickering L, Marciniak CM, et. al. Wii Fit balance board playing improves balance and gait in Parkinson disease. PM R. 2013; 5(9):769-77.

13. Pompeu JE, Arduini LA, Botelho AR, Fonseca MB, Pompeu SM, Torriani-Pasin C. Feasibility, safety and outcomes of playing Kinect Adventure for people with Parkinson's disease: a pilot study. Physiotherapy. 2014; 100(2):162-8

14. Esculier JF, Vaudrin J, Bériault P, Gagnon K, Tremblay LE. (2012). Home-based balance training programme using Wii Fit with balance board for Parkinsons's disease: a pilot study. J Rehabil Med. 2012; 44(2):14450 .

15. Bower KJ, Clark RA. McGinley JL. (2014). Clinical feasibility of the Nintendo Wii ${ }^{\mathrm{TM}}$ for balance training post-stroke: a phase II randomized controlled trial in an inpatient setting. Clin Rehabil. 2014; 28(9):912-23.

16. Pluchino A, Lee SY, Asfour S, Roos BA, Signorille JF. Pilot study comparing changes in postural control after training using a video game balance board program and 2 standard activity-based balance intervention programs. Arch Phys Med Rehabil. 2012; 93(7):1138-46.

17. Laver K, George S, Ratcliffe J, Quinn S, Whitehead C, Davies O, Crotty M. Use of an interactive video gaming program compared with conventional physiotherapy for hospitalised older adults: a feasibility trial. Disabil Rehabil. 2012; 34(21):1802-08.

18. Cho KH, Lee KJ, Song CH, . (2012). Virtual-reality balance training with a video-game system improves dynamic balance in chronic stroke patients. Tohoku J Exp Med. 2012; 228(1):69-74.

\section{CONFLITO DE INTERESSES}

Os autores declaram não haver conflitos de interesse.

\section{AUTOR PARA CORRESPONDÊNCIA}

\section{Yago Tavares Pinheiro}

yagotavares5@gmail.com

Submetido em 04/11/2016 Aceito em 05/12/2016 